\section{Investigação Morfológica e Estrutural de Nanopartículas Magnéticas do Tipo Core@Shell por Meio Técnicas de Radiação Síncrotron e Microscopia Eletrônica de Transmissão}

F. H. Martins ${ }^{\mathrm{a}, \mathrm{d}}$, V. Pilati ${ }^{\mathrm{a}}$, R. Cabreira-Gomes ${ }^{\mathrm{b}}$, F. L. O. Paula ${ }^{\mathrm{a}, \mathrm{d}}$, R. Aquino ${ }^{\mathrm{c}}$, R. Perzynski ${ }^{\mathrm{d}}$, and J. Depeyrot ${ }^{\mathrm{a}}$

${ }^{a}$ Grupo 3Nano, Instituto de Física, Universdade de Brasília, Brasília, Brasil. ${ }^{b}$ Departamento de Física, Universidade Federal de Santa Catarina, Florianópolis, Brasil. ${ }^{c}$ Grupo 3Nano, Faculdade de Planaltina, Universidade de Brasília, Brasília, Brasil. ${ }^{d}$ Laboratório Phenix, Sorbonne Universités CNRS, Paris, France.

\section{RESUMO}

A estrutura, a morfologia e o tamanho de nanopartículas de ferrita mista de $\mathrm{Zn}-\mathrm{Mn}$ obtidas durante o processo de síntese de fluidos magnéticos foram investigadas utilizando as técnicas de Espectroscopia de Raios X por Energia Dispersiva, Difração de Raios X, Microscopia de Transmissão Eletrônica e Difração de elétrons. Almejando a estabilidade química desses materiais em meio ácido, um tratamento de superfície promove o revestimento dessas partículas por uma fina camada de maguemita, o que nos permite classificar essas estruturas em um modelo core-shell. O resultado combinado do uso de várias técnicas de caracterização nos possibilita verificar que essas partículas são polidispersas, apresentam fortes tendências a formar aglomerados e a ocorrência de apenas planos cristalinos característicos de estruturas do tipo espinélio nos permite concluir que o tratamento de superfície imposto a esses materiais não modifica sua cristalinidade, tampouco promove o surgimento de materiais indesejados após o condicionamento de superfície.

\begin{abstract}
The structure, morphology, and size of mixed ferrite nanoparticles of $\mathrm{Zn}$-Mn, obtained during the synthesis process of a magnetic fluid were investigated combining the results obtained by several techniques such as Energy-Dispersive X-ray Spectroscopy, X-Ray Diffraction, Transmission Electron Microscopy, and Selected Area Electron Diffraction. Aiming to achieve the chemical stability of those materials in acid media, an empirical surface treatment promotes the coating of these particles with a thin layer of maghemite, which allows classifying these structures in a core-shell model. The results obtained from the characterization techniques shows that these particles are polydisperse, they present a strong tendency to agglomerate, and the occurrence of crystallographic planes only of the spinel structure allows us to conclude that the surface treatment imposed on those materials doesn't change their crystallinity, neither it promotes the appearance of unwanted materials after the surface conditioning.
\end{abstract}

\section{INTRODUÇÃO}

Nanocristais de ferrita são nanopartículas magnéticas (NPs) bem conhecidas por suas propriedades físicas e químicas peculiares [1-3]. Eles podem ser revestidos por diferentes compostos com o objetivo de aprimorar suas aplicações biomédicas [4,5] e tecnológicas [6,7] a que se propõe. Nesse sentido, eles podem ser descritos em uma estrutura core-shell, que consiste em um núcleo de ferrita recoberto por uma camada superficial. No caso particular de nanopartículas core-shell bi-magnéticas, é possível combinar diferentes fases magnéticas para enfatizar o comportamento global [8]. As propriedades físicas resultantes das NPs dependem fortemente de ambas estruturas (núcleo e superfície) e também da interface entre elas. Deste modo, um conhecimento profundo e satisfatório de tais propriedades se faz necessário e têm despertado bastante interesse na área da nanotecnologia. Neste contexto, as propriedades estruturais e morfológicas de nanopartículas de ferrita mista de $\mathrm{Zn}-\mathrm{Mn}$ obtidas durante o processo de síntese de fluidos magnéticos de dupla camada elétrica foram investigadas 
utilizando as técnicas de Espectroscopia de Raios X com Energia Dispersiva, Difração de Raios X, Microscopia de Transmissão Eletrônica e Difração de Elétrons.

\section{MATERIAIS E MÉTODOS}

As amostras investigadas neste trabalho são nanopartículas core-shell de ferritas mistas $\mathrm{Zn}_{\mathrm{y}} \mathrm{Mn}_{(1-\mathrm{y})} \mathrm{Fe}_{2} \mathrm{O}_{4} @ \gamma-\mathrm{Fe}_{2} \mathrm{O}_{3}$, chamadas NPs-CS (nanopartículas core-shell), obtidas pelo método de coprecipitação [9-13] durante a síntese de fluidos magnéticos EDL-MF (Electric Double Layered Magnetic Fluid - EDL-MF).

O processo de obtenção de um colóide magnético do tipo EDL-MF consiste resumidamente em três etapas que estão esquematicamente representadas no diagrama da Figura 1. Inicialmente as nanopartículas são sintetizadas [14] a partir da policondensação dos cátions $\mathrm{Fe}^{3+}, \mathrm{Zn}^{2+} \mathrm{e}$ $\mathrm{Mn}^{2+}$, em hidróxido de sódio $(\mathrm{NaOH} 2 \mathrm{~mol} / \mathrm{L})$, à temperatura de $100^{\circ} \mathrm{C}$ e sob agitação mecânica constante. A proporção dos cátions nas soluções foi de 1:y:(1-y), onde y é a proporção de zinco variando de 0,1 a 0,9 , objetivando a composição química nominal da ferrita $\mathrm{Zn}_{\mathrm{y}} \mathrm{Mn}_{(1-\mathrm{y})} \mathrm{Fe}_{2} \mathrm{O}_{4}$. Em seguida, foi realizado um tratamento de superfície (etapa 2) que inicia-se através de uma lavagem com ácido nítrico $\left(\mathrm{HNO}_{3} 2 \mathrm{~mol} / \mathrm{L}\right)$ para remoção de quaisquer subprodutos indesejáveis e inversão da carga superficial das nanopartículas (2a), seguido de tratamento hidrotérmico de superfície $(2 \mathrm{~b})$ com nitrato férrico $\mathrm{Fe}\left(\mathrm{NO}_{3}\right)_{3}(0,5 \mathrm{~mol} / \mathrm{L})$, por aproximadamente 15 minutos. Finalmente, na terceira etapa, as NPs-CS são dispersadas em meio aquoso através de um ajuste de força iônica, levando à obtenção de EDL-MF com estabilidade química a longo prazo em meio ácido. De acordo com o teor nominal de zinco na estrutura desses materiais, as amostras foram nomeadas de ZM1 a ZM9, onde, por exemplo a amostra ZM1 apresenta um teor nominal de zinco $\mathrm{y}=0,1$.

A estrutura morfoquímica das amostras aqui investigadas foi sondada recentemente [14] por técnicas modernas de microscopia de transmissão eletrônica. Na ocasião, foi utilizado um Espectroscópio de Raios X com Energia Dispersiva (EDX) acoplado a um Microscópio de Varredura e Transmissão Eletrônica para quantificar a composição química do núcleo e da superfície na estrutura das nanopartículas e verificou-se que todas as amostras apresentaram uma fina camada superficial de maguemita, resultado que comprova de fato uma estrutura do tipo core-shell destas NPs.

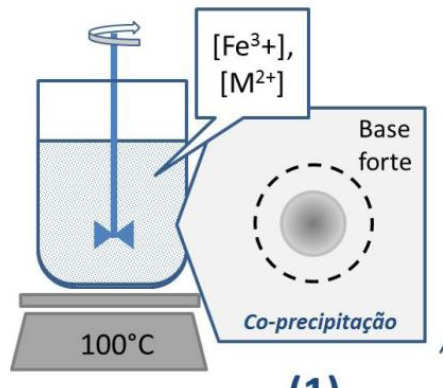

(1)

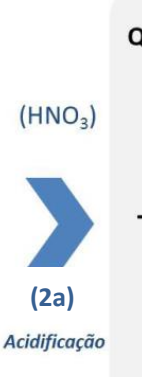

(2)

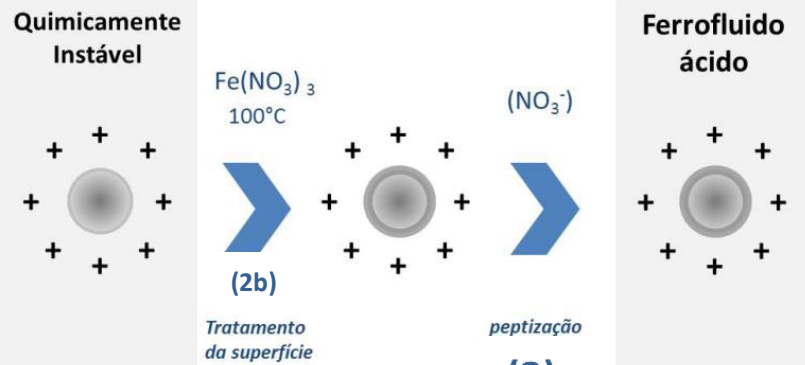

(3)

Figura 1: Esquema do processo de elaboração de um fluido magnético. Figura adaptada [15].

Para verificar a qualidade e a composição química dos materiais elaborados, foram realizadas medidas de EDX na Central de Análises do Instituo de Química da Universidade de Brasília - IQ/UnB. Esta técnica nos permite obter informações sobre as concentrações dos metais que constituem a estrutura da ferrita e a fração em metal divalente $\chi_{\mathrm{M}}$.

As NPs-CS foram submetidas a medidas de Difração de Raios $X$ com o intuito de investigar mudanças estruturais decorrentes da substituição de cátions de Mn por Zn. Os dados experimentais foram coletados na linha de luz XRD1 do Laboratório Nacional de Luz Síncrontron em Campinas/Brasil utilizando um feixe monocromático de luz de energia 6,99 keV (aproximadamente $1,77 \AA$ ).

Imagens de Microscopia de Transmissão Eletrônica foram adquiridas junto ao Service de Microscopie Electronique de l'UFR de Chimie do laboratório Phenix da Université Pièrre et 
Marie Curie (UPMC) Paris Sorbonne VI em Paris/França utilizando um microscópio do tipo JEOL mod. JEM $2010^{\odot}$ operado a $200 \mathrm{kV}$ com $\lambda=0,025 \AA$ para compreendermos a morfologia e a estrutura cristalina dos materiais aqui investigados.

\section{RESULTADOS E DISCUSSÃO}

As informações características das NPs-CS investigadas neste trabalho foram reunidas na Tabela 1. A partir das medidas de EDX pode-se obter a fração molar em metal divalente, a fração volumétrica da superfície e a espessura da camada superficial de maguemita rica em ferro dentro de um modelo core-shell.

A fração em metal divalente que corresponde a uma ferrita estequiométrica tem como valor ideal 0,33. Entretanto, observamos valores inferiores já após a coprecipitação, com perda de $\mathrm{Zn}^{2+}$ e enriquecimento em $\mathrm{Fe}^{3+}$, efeito que aumenta com o teor nominal de zinco y. Esses resultados devem-se a formação de ferritas não estequiométricas [14], o que ocorre graças ao $\mathrm{pH}$ fortemente alcalino [16]. Após o tratamento superficial, $\chi_{M}$ diminui ainda mais, evidenciando o enriquecimento em ferro na superfície das NPs-CS promovido pelo tratamento superficial. Em trabalhos anteriores [13] observou-se que o tratamento térmico em nitrato férrico além de promover um acréscimo na concentração de ferro acarreta também na remoção de cátions divalentes mal coordenados na superfície e ambos os fatores contribuem para a redução do valor de $\chi_{\mathrm{M}}$. Além disso, observa-se um progressivo aumento na fração volumétrica $\phi_{\mathrm{s}} / \phi$ e da espessura da camada superficial à medida que se diminui o valor de $\chi_{\mathrm{M}}$, mostrando que após o tratamento de superfície em nitrato férrico se faz necessário um modelo core-shell para compreensão da estrutura das amostras aqui examinadas.

Tabela 1: Características das NPs-CS de ferrita mista de Zn-Mn. $\chi_{M}$ é a fração molar em metal divalente antes (1) e depois do tratamento de superfície (2b), $\phi_{s} / \phi$ é a fração volumétrica da superfície, e é a espessura da camada superficial, $\langle a\rangle$ é o parâmetro de malha, $D_{r x}{ }^{\text {Sch }}$ e $D_{T E M}$ são respectivamente os diâmetros cristalinos obtidos por Difração de Raios X e Microscopia de

Transmissão Eletrônica e $\sigma e ́$ a polidispersão.

\begin{tabular}{ccccccccc}
\hline Amostra & $\begin{array}{c}\chi_{\mathbf{M}} \\
(\mathbf{1})\end{array}$ & $\begin{array}{c}\chi_{\mathbf{M}} \\
(\mathbf{2 b})\end{array}$ & $\boldsymbol{\phi}_{\mathbf{S}} / \boldsymbol{\phi}(\boldsymbol{\%})$ & $\boldsymbol{e}(\mathbf{n m})$ & $\begin{array}{c}\langle\boldsymbol{a}\rangle \\
(\mathbf{n m})\end{array}$ & $\begin{array}{c}\boldsymbol{D}_{\mathbf{r x}}^{\text {Sch }} \\
(\mathbf{n m})\end{array}$ & $\begin{array}{c}\boldsymbol{D}_{\text {TEM }} \\
(\mathbf{n m})\end{array}$ & $\boldsymbol{\sigma}$ \\
\hline ZM1 & $0,332(1)$ & $0,296(1)$ & - & - & $0,844(1)$ & $23,7(5)$ & $16,1(5)$ & $0,46(3)$ \\
ZM2 & $0,308(1)$ & $0,241(1)$ & 17,4 & $0,47(1)$ & $0,842(1)$ & $13,9(5)$ & $9,2(1)$ & $0,30(1)$ \\
ZM3 & $0,303(1)$ & $0,220(1)$ & 25,7 & $0,57(1)$ & $0,839(1)$ & $11,1(5)$ & $8,8(2)$ & $0,39(2)$ \\
ZM4 & $0,300(1)$ & $0,211(1)$ & 29,3 & $0,60(1)$ & $0,840(1)$ & $10,1(5)$ & $6,2(1)$ & $0,38(2)$ \\
ZM5 & $0,277(1)$ & $0,212(1)$ & 22,2 & $0,46(1)$ & $0,840(1)$ & $10,5(5)$ & $7,4(1)$ & $0,35(1)$ \\
ZM6 & $0,279(1)$ & $0,201(1)$ & 27,9 & $0,47(1)$ & $0,838(2)$ & $8,4(5)$ & $5,9(1)$ & $0,38(1)$ \\
ZM7 & $0,245(1)$ & $0,180(1)$ & 27,1 & $0,51(1)$ & $0,838(2)$ & $9,3(5)$ & $4,5(1)$ & $0,36(1)$ \\
ZM8 & $0,285(1)$ & $0,177(1)$ & 41,2 & $0,80(1)$ & $0,840(2)$ & $9,1(5)$ & $4,9(1)$ & $0,32(1)$ \\
ZM9 & $0,249(1)$ & $0,158(1)$ & 42,3 & $0,72(1)$ & $0,838(3)$ & $7,8(5)$ & $5,3(1)$ & $0,41(1)$ \\
\hline
\end{tabular}

Os difratogramas de Raios X de todas as amostras (ZM1-ZM9) são apresentados na Figura 2a. Podemos ver que os picos de difração são largos devido ao tamanho manométrico do material analisado. Utilizando a equação da lei de Bragg e a equação de simetria cúbica podemos indexar os picos de difração e, comparando os resultados com aqueles apresentados pelo Centro Internacional de Dados de Difração (ICDD n ${ }^{\circ}$ 98-002-8512 até -8516), podemos concluir que a estrutura desses materiais corresponde à estrutura do tipo espinélio [14, 17]. Além disso, uma análise direta da Figura 2 a nos permite concluir que a progressiva substituição de cátions de Mn por cátions de Zn não provoca mudanças na estrutura dos materiais e não há a ocorrência de picos que não correspondam à estrutura do espinélio, ou seja, à medida que se adiciona mais cátions de $\mathrm{Zn}$, estes são incorporados na estrutura da ferrita, fazendo com que o material resultante permaneça com a estrutura correspondente à ferrita espinélio [18].

Ainda se valendo da equação de Bragg e da equação de simetria cúbica, podemos determinar analiticamente uma média para o parâmetro de malha e estimar o tamanho cristalino das amostras através da equação de Scherrer [19] tomando a largura a meia altura do pico (311). 


\section{$10^{\text {th }}$ Workshop of Applied Crystallography \\ UFES, Vitória - ES, Brasil, May 2020}

Os valores foram coletados na Tabela 1. Verifica-se que os parâmetros de malha das NPs-CS não correspondem àqueles das ferritas bulk para qualquer teor de zinco na estrutura da ferrita. Essa diferença pode ser explicada pelo fato de que as amostras apresentam uma camada superficial de maguemita o que causa uma desordem estrutural e, também, pela competição dos seguintes fatores concorrentes entre si - perda de cátions de $\mathrm{Zn}^{2+}$ para a síntese hidrotérmica, oxidação dos cátions de $\mathrm{Mn}^{2+}$ para $\mathrm{Mn}^{3+}$ e $\mathrm{Mn}^{4+}$, forte afinidade química que alguns cátions apresentam em se alocarem em determinados interstícios e distribuição metaestável dos cátions em nanoescala [14-17]. Em se tratando do tamanho cristalino, podemos verificar que o aumento da concentração de zinco na estrutura da ferrita ocasionou uma diminuição do tamanho das NPs-CS, fato também reportado em outros trabalhos $[16,20]$.
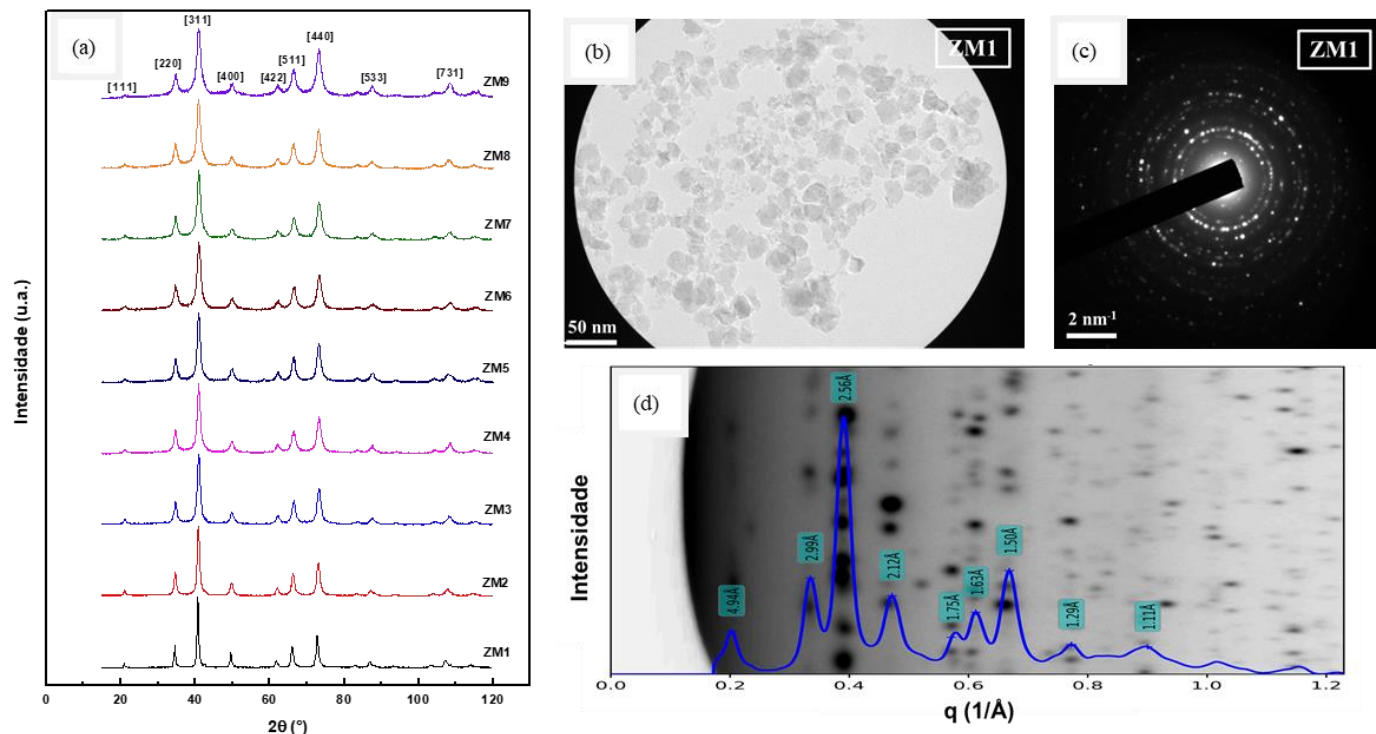

Figura 2: (a) Difratogramas de Raios X das amostras de NPs-CS de ferrita mista de Zn-Mn, (b) área selecionada para a realização da medida de difração de elétrons da amostra ZM1 com

$10 \%$ de teor de zinco, (c) padrão bidimensional obtido por difração eletrônica da região selecionada para a amostra ZMI e (d) difratograma eletrônico obtido através das intensidades distribuídas radialmente para a amostra ZMI.

A Figura 3 apresenta a microscopia da amostra ZM1. Uma análise qualitativa desta Figura nos permite concluir que a maioria das partículas observadas apresenta formato aproximadamente arredondado e, além disso, nota-se que as nanopartículas são homogêneas de modo que não são observados buracos ou descontinuidade. A ausência de subprodutos das etapas de síntese corrobora os resultados obtidos pela análise dos difratogramas de Raios X da Figura 2a. Ainda, não são observadas cadeias de

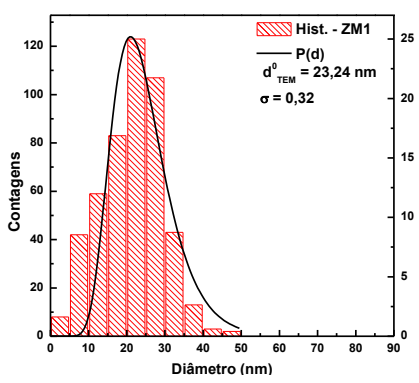

Figura 4: Ajuste da frequência de diâmetros em função da densidade de probabilidade do tipo Lognormal da micrografia da

Figura 3.

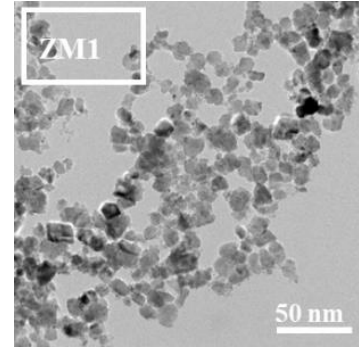
nanopartículas, entretanto podemos observar que elas têm forte tendência a se aglomerarem provavelmente devida à magnetização apresentada pelo material.

Figura 3: Micrografia da amostra de ferrita mista ZM1. Além de podermos visualizar as nanopartículas e reconhecer suas morfologias, é possível estimar o diâmetro cristalino médio $D_{T E M} \mathrm{e}$ determinar o tipo de distribuição de diâmetros que esses materiais apresentam.

Desta maneira, são realizadas medidas de diâmetro de aproximadamente 500 partículas não aglomeradas ou de espécimes cujo limite do grão seja suficientemente visível para determinar seu tamanho. Para todas as amostras investigadas, os histogramas obtidos desta análise estatística são semelhantes aos apresentados 
na Figura 4 e mostram que existe um intervalo de tamanho no qual concentra-se grande parte dos nanocristais, caracterizando desta forma uma larga distribuição em tamanho. Os valores obtidos para o diâmetro médio $D_{T E M}$ e para polidispersão $\sigma$ são apresentados na Tabela 1. A variação de $D_{\text {ТЕ }}$ em função do teor de Zn nas amostras de NPs-CS de ferrita mista apresenta o mesmo comportamento reportado pela análise do diâmetro obtido pela técnica de Difração de Raios X.

Podemos constatar a cristalinidade desses materiais utilizando o método de Difração de Elétrons de Área Selecionada - SAED. As Figuras 2b-d apresentam as etapas realizadas para o processamento dos padrões bidimensionais obtidos por SAED que envolvem a seleção da área, aquisição e tratamento dos dados obtidos. O programa Diffraction Ring Profiler é utilizado para realizar o processamento digital que consiste na indexação dos anéis existentes na Figura $2 \mathrm{c}$ e na conversão em uma distribuição radial apresentada na Figura 2d. O difratograma resultante possui uma dependência em $q$ (vetor de onda) onde as posições dos picos estão relacionadas com as distâncias interplanares $\left(d_{h k l}\right)$. Os padrões bidimensionais das amostras de NPs-CS de ferrita mista de $\mathrm{Zn}-\mathrm{Mn}$ com variados teores de $\mathrm{Zn}$ apresentam bastante similaridade com aquele apresentado

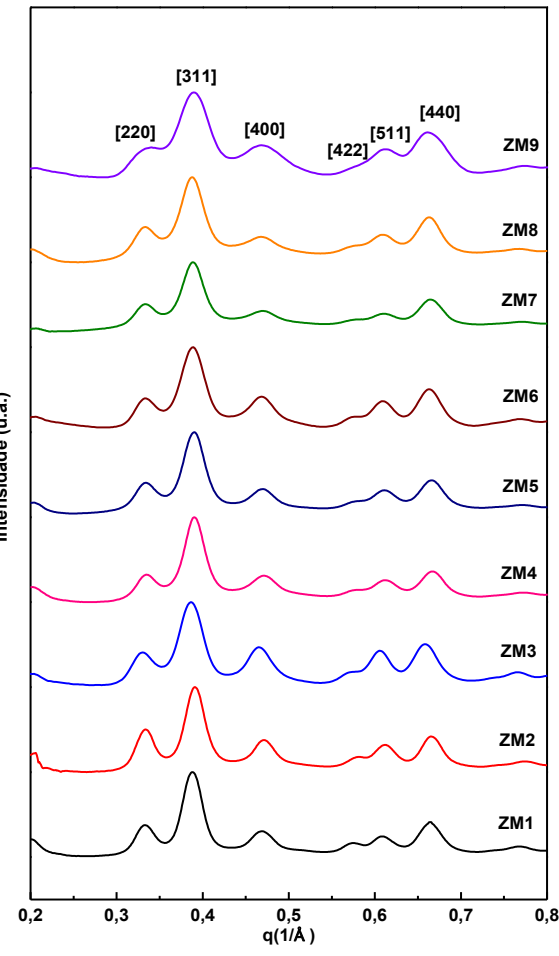

Figura 5: Difratogramas eletrônicos das amostras de NPsCS de ferrita de $\mathrm{Mn}-\mathrm{Zn}$. na Figura 2c.

Os difratogramas eletrônicos obtidos após o processamento dos padrões 2D são apresentados na Figura 5. É possível observar que, para todas as amostras, os picos coincidem entre eles nas mesmas posições o que nos leva a concluir que todas as amostras possuem o mesmo tipo de simetria e estrutura.

\section{CONCLUSÃO}

Neste trabalho investigamos a morfologia e a estrutura de nanopartículas core-shell de ferrita mista de Zn-Mn a partir da combinação dos resultados obtidos por várias técnicas experimentais, tais como Espectroscopia de Raios X com Energia Dispersiva, Difração de Raios X, Microscopia de Transmissão Eletrônica e Difração de Elétrons.

Observa-se que o processo de elaboração desses materiais, que leva em consideração um tratamento de superfície em nitrato férrico, promove o revestimento dessas partículas por uma camada superficial de maguemita. À medida que mais átomos de $\mathrm{Zn}$ são incorporados na estrutura cristalina das nanopartículas, observou-se uma redução da fração em metal divalente e um correspondente aumento da espessura da camada superficial. Além disso, verificou-se que o tratamento hidrotérmico de superfície não foi capaz de alterar a simetria do material investigado, entretanto, a estrutura das nanopartículas se modifica pela incorporação da camada superficial e passa a ser mais bem descrita em um modelo core-shell.

Medidas de Difração de Raios $X$ constataram que os parâmetros de malha não correspondem aos valores padrões apresentados nas tabelas ICDD devido, dentre outros fatores, à presença da camada superficial de maguemita, à oxidação dos cátions de manganês e ao enriquecimento em $\mathrm{Fe}^{3+}$ consequente da perda de $\mathrm{Zn}^{2+}$ durante a coprecipitação.

A morfologia e cristalinidade das amostras foram investigadas utilizando as técnicas de Microscopia de Transmissão Eletrônica e Difração de elétrons. Observou-se que as nanopartículas são homogêneas, levemente arredondadas e apresentam forte tendência a formarem aglomerados devido ao caráter magnético dos materiais. A partir da obtenção de histogramas, foi possível determinar os diâmetros das nanopartículas, que apresentaram bastante 
similaridade com aqueles determinados através da técnica de Difração de Raios X. As análises dos padrões 2D obtidos por Difração de Elétrons corroboram os resultados de XRD mostrando que a estrutura dos materiais corresponde à estrutura do tipo espinélio e que o processo de síntese não culminou na produção de subprodutos indesejados com estrutura e simetria diferente dos materiais aqui investigados, mostrando, assim, excelência na produção desses materiais.

\section{REFERÊNCIAS}

[1] Gubin, S. O., Magnetic Nanoparticles, Wiley-VCH Verlag GmbH \& Co. KgaA, (2009).

[2] Vollath, D., Nanomaterials, Wiley-VCH Verlag GmbH \& Co. KgaA, (2013).

[3] Kumar, N. e Kumbhat, S., Essentials in Nanoscience \& Nanotechnology, John Wiley \& Sons, Inc., (2016).

[4] Goya, G. F., Grazu, V., e Ibarra, M. R., Curr. Nanosci., 4, 1, (2008).

[5] Carta, D., Casula, M. F., Floris, P., Falqui, A., Mountjoy, G., Boni, A., Sangregorio, C., e Corrias, A., Phys. Chem. Chem. Phys., 12, 5074, (2010).

[6] Ivanets, A. I., Srivastava, V., Roshchina, M.Y., Sillanpää, M., Prozorovich, V. G. e Pankov, V.V., Ceram. Int., 44, 9097, (2018).

[7] Silva, F. G. D., Depeyrot, J., Campos, A. F. C., Aquino, R., Fiorani, D. e Peddis, D., J. Nanosci Nanotechnol., 19, 4888, (2019).

[8] Cabreira-Gomes, R., Silva, F. G., Aquino, R., Bonville, P., Tourinho, F. A., Perzynski, R. e Depeyrot, J., J. Magn. Magn. Mater., 368, 409, (2014).

[9] Gomes, J. A., Azevedo, G. M., Depeyrot, J., Mestnik-Filho, J., Paula, F. L. O., Tourinho, F. A.e Perzynski, R., J Phys. Chem. C, 116, 24281, (2012).

[10] Tourinho, F. A., Franck, R. e Massart, R., J. Mater. Sci., 25, 3249, (1990).

[11] Tourinho, F. A., Franck, R., Massart, R. e Perzynski, R., Progr. Colloid Polym Sci., 79:128, (1989).

[12] Aquino, R., Depeyrot, J., Sousa, M. H., Tourinho, F. A., Dubois, E. e Perzynski, R., Phys Rev. B., 72, 184435, (2005).

[13] Gomes, J. A., Sousa, M. H., Tourinho, F. A., Aquino, R., da Silva, G. J., Depeyrot, J., Dubois, E. e Perzynski, R., J. Phys. Chem. C, 112, 6220, (2008).

[14]Pilati, V., Cabreira-Gomes, R., Gomide, G. S., Coppola, P., Silva, F. G., Paula, F. L. O., Perzynski, R., Goya, G. F., Aquino, R. e Depeyrot, J., J. Phys. Chem. C, 122, 3028, (2018).

[15] Cabreira-Gomes, R. Dispersões e nanopartículas magnéticas do tipo core-shell. Tese (Doutorado) - Programa de Cotutela entre as Universidades UPMC e UnB, Paris/Brasília, (2014).

[16] Rath, C., Sahu, K. K., Anand, S., Date, S. K., Mishra, N. C., e Das, R. P., Journal of Magnetism and Magnetic Materials. 202, 77-84 (1999).

[17] Martins, F. H., da Silva, F. G., Paula, F. L.O., Gomes, J. A., Aquino, R., Mestnik-Filho, J., Bonville, P., Porcher, F., Perzynski, R. e Depeyrot, J., J. Phys. Chem. C, 121, 8982, (2017).

[18] Mamiya, H., Terada, N., Kitazawa, H., Hoshikawa, A., e T. Ishigaki, Journal of Magnetics, 16(2), 134-139 (2011).

[19] Scherrer, P., Nachr. Ges. Wiss. Göttingen. 26, 98 (1918).

[20] Auzans, E., Zins, D., Blums E., e Massart, R., Journal of Materials Science, 34: 1253-1260, (1999).

Agradecimentos: Este trabalho foi apoiado pelas agências CNPq, CAPES e FAP/DF. Agradecemos ao programa bilateral entre as universidades francesas e brasileiras UPMC/França e UnB/Brasil através do contrato CAPES/COFECUB no 714/11 e PICS/CNRS n 5939/11. Os 
autores também agradecem ao Serviço de Microscopia Eletrônica da UPMC e ao LNLS pelo período concedido para realizar medidas na linha de luz D12A-XRD1 de difração de Raios X. 